\title{
Radiation Dose and Image Quality in Pediatric Neck CT
}

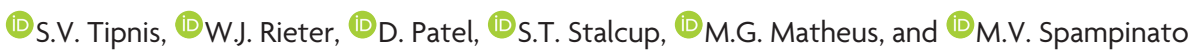

\begin{abstract}
BACKGROUND AND PURPOSE: Optimization of pediatric neck CT protocols is of critical importance in order to maintain good diagnostic image quality while reducing the radiation burden. Our aim was to evaluate the image quality of pediatric neck CT studies before and after the implementation of a low radiation dose protocol.
\end{abstract}

MATERIALS AND METHODS: We retrospectively reviewed 179 pediatric neck CT studies, 75 before and 104 after the implementation of low-dose protocols, performed in children $0-16$ years of age. The 2 cohorts were divided into 3 age groups, 0-4, 5-9, and 10-16 years. The signal-to-noise ratio was calculated using the axial image through the true vocal folds. Three neuroradiologists assessed the image quality of the same CT scan using a 5-point scoring system. We compared the CT dose index volume, dose-length product, image-quality ratings, and SNR of studies conducted at baseline and with low-dose protocols.

RESULTS: Image-quality ratings were lower in the low-dose than in the baseline cohort in children 10-16 years of age, but not in children $0-4$ and 5-9 years of age. The SNR was lower in the low-dose cohort than in the baseline cohort in children 0-4 and 10-16 years of age, but not in children 5-9 years of age. Despite the decrease in image-quality scores in older children, $97 \%$ of the studies (73/75) in the baseline cohort and $96 \%$ of studies (100/104) in the low-dose cohort were considered of sufficient image quality.

CONCLUSIONS: Images acquired with the low-dose CT protocols were deemed to be of sufficient quality for making a clinical diagnosis. Our initial results suggest that there may be an opportunity for further radiation dose reduction without compromising diagnostic image quality using iterative reconstruction algorithms.

ABBREVIATIONS: $\mathrm{CTDI}_{\mathrm{vol}}=\mathrm{CT}$ dose index volume; $\mathrm{DLP}=$ dose-length product

$\mathbf{P}$ ediatric CT volume has rapidly increased in the United States since the introduction of helical and multidetector CT, which allow fast image acquisition and a decreased need for moderate sedation. ${ }^{1-5}$ Because of the smaller size of the patients and higher radiation sensitivity, pediatric $\mathrm{CT}$ poses some unique challenges in terms of achieving diagnostic-quality images with the least possible radiation burden to the patients. ${ }^{4,6-12}$ Optimization of a pediatric neck CT protocol is of utmost importance because in this study, the thyroid gland, one of the most radiosensitive organs, is

Received December 19, 2018; accepted after revision April 18, 2019.

From the Department of Radiology and Radiological Science (S.V.T., W.J.R., D.P., S.T.S., M.G.M., M.V.S.) and Center for Biomedical Imaging (M.V.S.), Medical University of South Carolina, Charleston, South Carolina.

Paper previously presented at: American Society of Neuroradiology Annual Meeting and the Foundation of the ASNR Symposium, June 2-7, 2018; Vancouver, British Columbia, Canada.

Please address correspondence to Maria Vittoria Spampinato, MD, Department of Radiology and Radiological Science, Medical University of South Carolina, 96 Jonathan Lucas, MSC 323, Charleston, SC 29425; e-mail: spampin@musc.edu;

@mvspampinato

http://dx.doi.org/10.3174/ajnr.A6073 directly exposed to the x-ray beam. ${ }^{13-15}$ Thus, it is critical that the examination be clinically indicated, with a high benefit-to-risk ratio. All CT examinations should be optimized to yield diagnostic images at the lowest possible radiation dose, following the principle of as low as reasonably achievable (ALARA).

We conducted a departmental quality-improvement project to optimize pediatric neck CT protocols. The goal of this study was to minimize the radiation exposure to patients while maintaining the required diagnostic image quality. This was done by reviewing and scoring baseline scans acquired with unoptimized CT protocols; applying appropriate dose-reduction criteria including age-stratification to optimize the image acquisition; and finally reviewing and scoring the images in the follow-up cohort to confirm their diagnostic value.

\section{MATERIALS AND METHODS}

This study was approved by the institutional review board with a waiver of informed consent. The patient population included a baseline cohort and a follow-up cohort that underwent neck 
Table 1: Total number of patients and distribution of patient age and sex in the 3 age groups of the study

\begin{tabular}{|c|c|c|c|c|}
\hline Group & $0-4 \mathrm{yr}$ & $5-9 \mathrm{yr}$ & $10-16 \mathrm{yr}$ & $0-16$ yr \\
\hline \multicolumn{5}{|l|}{ Baseline cohort } \\
\hline o & 10 & 11 & 20 & 41 \\
\hline q & 7 & 7 & 20 & 34 \\
\hline Total (No.) & 17 & 18 & 40 & 75 \\
\hline \multicolumn{5}{|c|}{ Follow-up cohort } \\
\hline 0 & 17 & 17 & 21 & 55 \\
\hline q & 8 & 9 & 32 & 49 \\
\hline Total (No.) & 25 & 26 & 53 & 104 \\
\hline
\end{tabular}

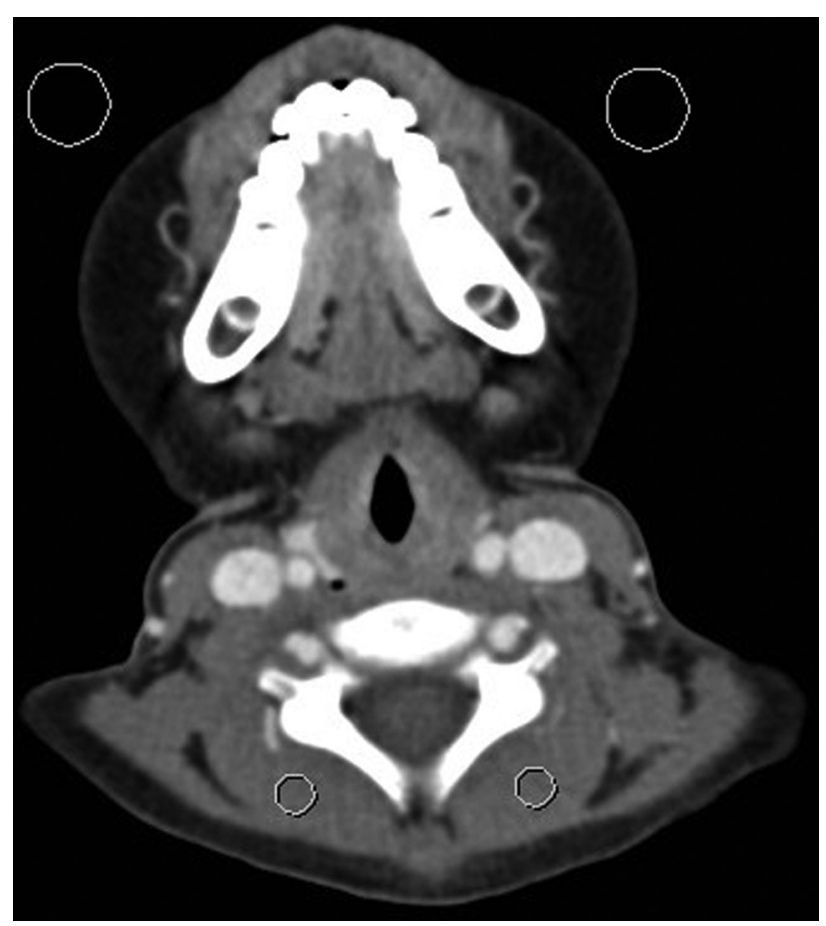

FIG 1. Representative CT image of a 6-year-old boy at the level of the vocal cords for assessment of the signal-to-noise ratio. Background noise was estimated as the average SD in Hounsfield units of 2 ROIs drawn over the air anterior to the patient (large circles). Signal was estimated as the average attenuation in Hounsfield units of 2 ROls drawn over the paraspinal musculature (small circles).

CT examinations before and after the implementation of optimized, age-specific, pediatric neck CT protocols at our institution.

\section{Patient Population}

Both the baseline and the follow-up cohorts in the study were identified by searching the PACS. Because of the differences in the body habitus of the patient population between 0 and 16 years of age, we thought it necessary to divide each cohort into 3 age groups to make a proper comparison. Accordingly, the study compared the examinations between the 2 cohorts in 3 distinct subgroups of $0-4,5-9$, and 10-16 years of age. We used the age-classification scheme for pediatric CT protocols proposed by Paolicchi et al. ${ }^{16}$ We changed the age range for the older age group from 10-14 years to 10-16 years because at our institution, we use pediatric neck CT protocols in patients 16 years of age or younger.

For the baseline cohort, we retrospectively reviewed all neck CT examinations acquired at our institution in patients 16 years
Table 2: Interpretation of the subjective image-quality scores for the individual, prelabeled CT image slices at the level of the vocal cords

\begin{tabular}{ll}
\hline Score & Comment \\
\hline 1 & $\begin{array}{c}\text { Image quality very poor (significant noise and/or } \\
\text { artifacts, study uninterpretable) }\end{array}$ \\
2 & Image quality poor (noise and/or artifacts, can only \\
& answer broad clinical questions) \\
4 & Image quality adequate (some noise and/or artifacts, \\
& but study interpretable) \\
& above-average image quality (minimal noise and/or \\
5 & artifacts) \\
& of artifacts)
\end{tabular}

of age or younger between May 2013 and April 2014. Scans acquired as part of radiation planning, studies for which the radiation dose summary sheet was not available on the PACS, those conducted without the use of automatic tube current modulation, and those with significant artifacts due to motion or hardware were excluded from the study. Thus, a total of 75 pediatric neck $\mathrm{CT}$ examinations were included in the baseline cohort. For the follow-up cohort, we retrospectively reviewed all neck CT examinations acquired at our institution in patients 16 years of age or younger between February 2016 and January 2017 using the modified, reduced-dose, and age-specific acquisition protocols. With the same exclusion criteria used for the baseline cohort, we selected a total of 104 pediatric neck CT examinations for the follow-up cohort. Table 1 shows the distribution of patients in the 2 cohorts and the 3 age groups.

\section{Image Acquisition}

All neck CT examinations were helical acquisitions from the forehead to the thoracic inlet and were acquired with tube current modulation on multidetector CT scanners (Somatom Sensation 64, Somatom Definition 64, or Somatom Definition Flash; Siemens, Erlangen, Germany). The scans were obtained using the manufacturer-provided automatic tube current modulation algorithms (CARE Dose4D; Siemens), with a pitch of 0.8-1.2 depending on the scanner, and using a display FOV ranging from 13 to $25 \mathrm{~cm}$. Scans were obtained following administration of a nonionic iodinated contrast agent $(2 \mathrm{~mL} / \mathrm{kg}$ of body weight $)$ and following a 90-second delay from the start of the contrast injection. Demographic data (age and sex) were obtained for each patient. Acquisition parameters, including the kilovolt, milliampere-seconds, and CT dose index volume $\left(\mathrm{CTDI}_{\mathrm{vol}}\right.$ ) (corresponding to a $32-\mathrm{cm}$ acrylic dosimetry phantom) were obtained from dose-report sheets imported into the PACS. Note that the vendor software uses the $32-\mathrm{cm}$ phantom for all head and neck scans by default while using the $16-\mathrm{cm}$ phantom for the head scans. We believe that the inclusion of the shoulder region in the scans is the primary reason for using the $32-\mathrm{cm}$ phantom for the head and neck scans, as opposed to the $16-\mathrm{cm}$ phantom (which is used in the head scans alone).

Subjective and quantitative image-quality assessment (described in detail the following 2 sections) was conducted by 3 board-certified neuroradiologists for the baseline cohort. Most studies (97\%) in the baseline cohort were deemed of diagnostic quality by the reviewers. On the basis of these data, we adjusted 
Table 3: Median values of the scan parameters, signal-to-noise ratio, image-quality scores, estimated effective dose, and the percentage difference for the 2 cohorts

\begin{tabular}{|c|c|c|c|c|c|c|c|c|c|}
\hline \multirow[b]{2}{*}{ Parameter } & \multicolumn{3}{|c|}{$0-4$ yr } & \multicolumn{3}{|c|}{$5-9 \mathrm{yr}$} & \multicolumn{3}{|c|}{$10-16 \mathrm{yr}$} \\
\hline & I & II & \% Diff & I & II & \% Diff & I & II & \% Diff \\
\hline Tube current (mAs) & 150 & 128 & -15 & 182 & 162 & -11 & 266 & 165 & -38 \\
\hline $\mathrm{CTDI}_{\mathrm{vol}}(\mathrm{mGy})$ & 7.4 & 4.4 & -41 & 8.6 & 5.5 & -36 & 20 & 7 & -67 \\
\hline Scan length (cm) & 17.6 & 17.8 & 1 & 20.3 & 21 & 5 & 26 & 27 & 1 \\
\hline $\mathrm{DLP}$ (mGy-cm) & 125 & 77 & -38 & 175 & 132 & -24 & 522 & 240 & -54 \\
\hline $\mathrm{ED}(\mathrm{mSv})$ & 2.1 & 1.2 & -40 & 2.2 & 1.7 & -21 & 9.7 & 4.4 & -55 \\
\hline SNR & 16.4 & 13.4 & -18 & 13.4 & 16.3 & 22 & 20.2 & 16.2 & -20 \\
\hline IQ & 3.67 & 4.00 & 9 & 4.00 & 4.00 & 0 & 4.33 & 4.00 & -8 \\
\hline
\end{tabular}

Note:-IQ indicates image quality; ED, effective dose; Diff, difference; I, baseline; II, follow-up.

$\square$ Baseline ש Followup

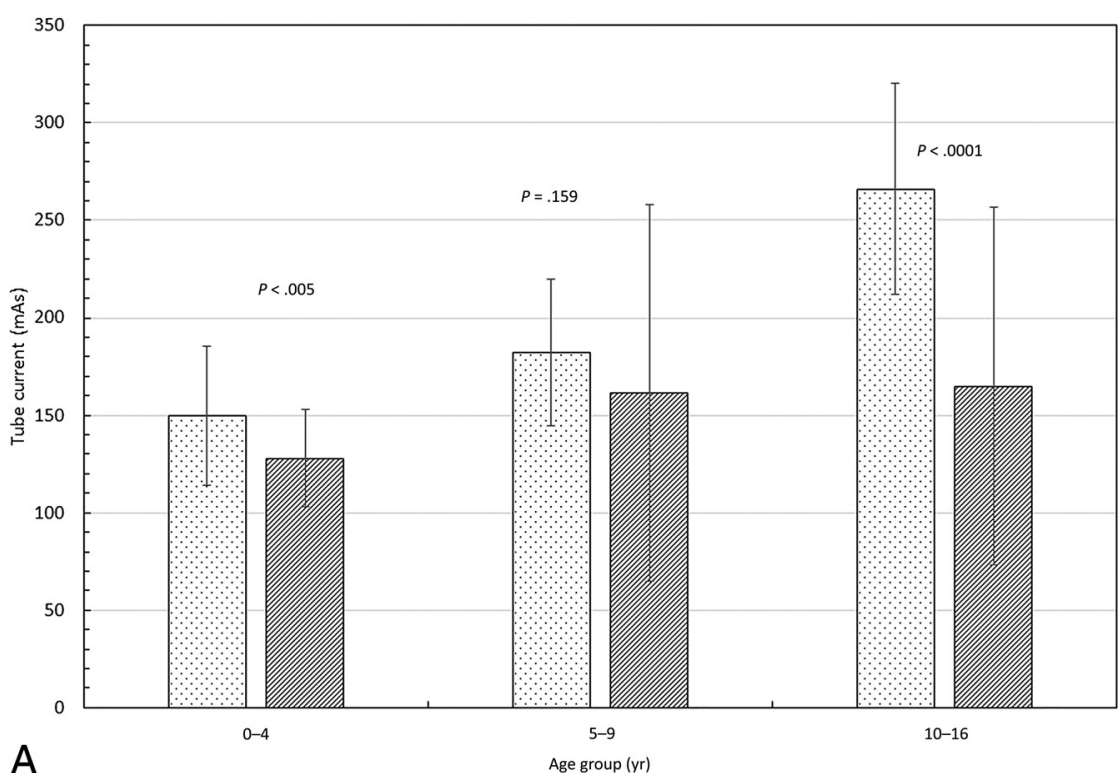

A

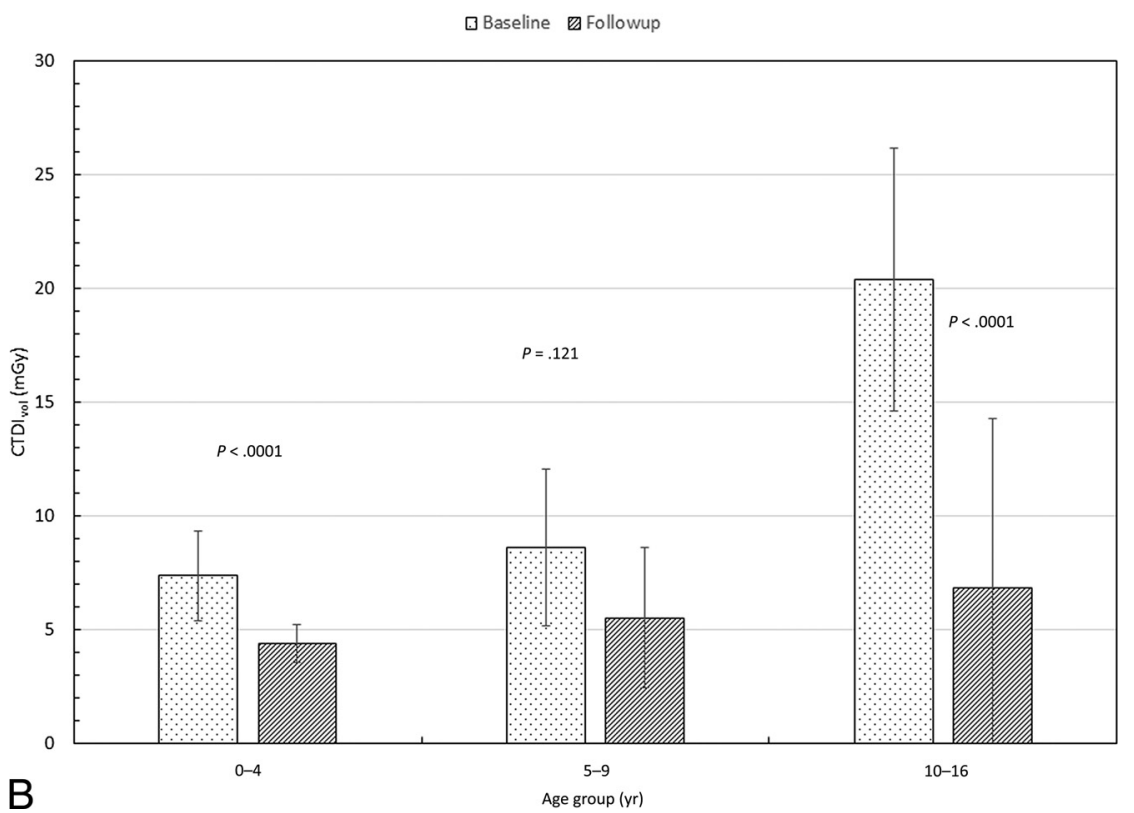

FIG 2. Comparison of the median values of tube current (milliampere-seconds) ( $A$ ), radiation output of scanner $\left(C T D I_{\text {vol }}\right)(B)$, and scan length between the 2 cohorts $(C)$. Also shown are $P$ values from an unpaired $t$ test.

the target $\mathrm{CTDI}_{\mathrm{vol}}$ to lower values for the follow-up cohort. Accordingly, the target $\mathrm{CTDI}_{\mathrm{vol}}$ values for the follow-up cohort were set at 6,8 , and $15 \mathrm{mGy}$ for the $0-4,5-9$, and $10-16$ age groups, respectively, by adjusting the reference milliampere-seconds for the examination protocols. These values corresponded to the first-quartile $\mathrm{CTDI}_{\mathrm{vol}}$ for each age group from the baseline cohort. Our rationale for this choice was that the first quartile (25th percentile) of the CTDI ${ }_{\text {vol }}$ represents a reasonable compromise between radiation dose and diagnostic quality.

\section{Subjective Assessment of Image Quality}

From each neck CT examination, 1 image at the level of the true vocal cords was selected for evaluation (Fig 1). Three board-certified neuroradiology attending physicians subjectively assessed the image quality of each selected image. For the follow-up cohort, the same 3 board-certified neuroradiologists assessed the image quality of each selected image. Readers were shown the complete image set on a standard reading room workstation in a randomized manner and were blinded to patient demographics, study indications, and protocol parameters during the rating process. Readers were allowed to change window and level settings as in routine image interpretation and were instructed to assign an integer score ranging from 1 to 5 using the rating system depicted in Table 2. Scores were based on diagnostic-quality parameters such as image noise, intrinsic artifacts, and contrast among tissues. Scores of $\geq 3$ were considered of sufficient quality for clinical diagnosis. Subjective imagequality interrater agreement among the 3 readers was assessed with the intraclass correlation coefficient using the Statistical Package for the Social Sciences software (Version 22; IBM, Armonk, New York). The intraclass correlation coefficient was interpreted as follows: $0-0.2$, poor agreement; $0.3-0.4$, fair agreement; $0.5-0.6$, moderate agreement; $0.7-0.8$, strong agreement; and $>0.8$, almost perfect agreement. 
$\square$ Baseline Followup

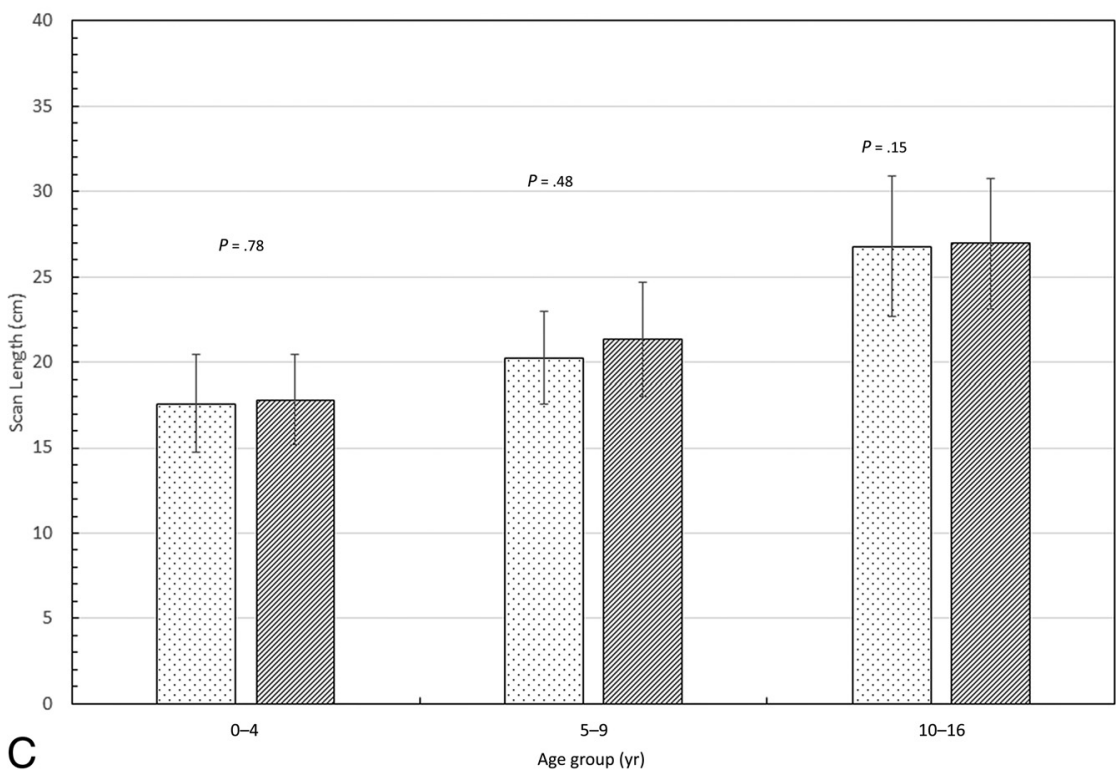

FIG 2. Continued.

\section{Quantitative Assessment of Image Quality}

For each patient in the study group, a quantitative assessment of image quality was performed by measuring the image background noise $(\mathrm{BN})$ and calculating the signal-to-noise ratio. The BN was defined as the average SD in Hounsfield units of 2 ROIs measuring $\sim 1.0 \mathrm{~cm}^{2}$ drawn over the air anterior to the patient (Fig 1) on the neck CT image obtained at the level of the true vocal cord. ${ }^{17,18}$ Image signal $\left(\mathrm{M}_{\mathrm{HU}}\right)$ was defined as the average attenuation of 2 ROIs measuring $\sim 0.5 \mathrm{~cm}^{2}$ drawn over the paraspinal musculature. The SNR was then calculated as

$$
\mathrm{SNR}=\mathrm{M}_{\mathrm{HU}} / \mathrm{BN} \text {. }
$$

We believe that the SNR is a more reproducible metric than the contrast-to-noise ratio in pediatric neck CT due to a lack of consistency across subjects with respect to pathology, fat content, and the degree of contrast opacification in the internal jugular vein.

\section{Effective Dose}

Finally, to estimate the effective dose for the patient cohort, we used the data published by Deak et $\mathrm{al}^{19}$ in the following manner: This publication lists the age-specific " $k$ " conversion factors for neck CT examinations for neonates; 1, 5, 10 years of age; and adults. The authors used mathematically defined phantoms with the most recent International Commission on Radiological Protection Publication 103 tissue-weighting factors and Monte Carlo simulations to estimate organ and effective doses from multislice CT examinations. We used a polynomial fit and interpolated these data to estimate the appropriate $\mathrm{k}$ value for the median age for each of our groups because the article has conversion factors specified only for neonates; children 1, 5, and 10 years of age; and adults. Note that we did not use the factors to estimate doses for individual patients, but only for a "median" patient of each age group/ cohort in the study.

\section{RESULTS}

Subjective image-quality interrater agreement was assessed for the baseline cohort as well as for the follow-up cohort. There was strong agreement among the 3 neuroradiology attending physicians for the baseline cohort (intraclass correlation coefficient $=$ 0.769 ; 95\% confidence interval, $0.676-$ 0.839; $P<.001)$ and for the follow-up cohort (intraclass correlation coefficient $=0.695 ; 95 \%$ confidence interval, $0.567-0.790 ; P<.001)$. Scores of all the readers were averaged for each CT image in the baseline cohort and the follow-up cohort.

Table 3 shows the median values of milliampere-seconds, CTDI ${ }_{\mathrm{vol}}$, scan length, dose-length product (DLP), effective dose, SNR, and image quality by age groups for the baseline and follow-up patient cohorts. Also shown is the percentage difference among the values of these parameters between the 2 cohorts. For convenience, these values are also depicted graphically in Figs 2-4, with $P$ values from an unpaired $t$ test to examine the significance of the difference between the 2 cohorts. Error bars in the figures represent 1 SD. Ninety-seven percent of the studies $(73 / 75)$ in the baseline cohort and 96\% (100/104) in the follow-up cohort were considered of sufficient image quality.

Tube current, CTDI ${ }_{\mathrm{vol}}$, and DLP were significantly lower in the follow-up cohort than in the baseline cohort in children $0-4$ and $10-16$ years of age, but not in children 5-9 years of age (Figs 2 and 3). Scan length did not differ between the baseline and follow-up cohorts in any age group (Fig 2). The SNR was lower in the low-dose than in the baseline cohort in children $0-4$ and $10-16$ years of age, but not in children 5-9 years of age (Fig 4). Image-quality ratings for all 3 age groups did not show major differences; they were lower in the follow-up cohort than in the baseline cohort in children 10-16 years of age, slightly higher in children $0-4$ years of age, and with no significant difference for those 5-9 years of age (Fig 4). Despite the decrease in image-quality scores in older children, in the follow-up patient cohort, $96 \%$ of the studies were considered of sufficient image quality (average image quality, $\geq 3$ ). Comparison of the effective doses before and after the implementation of low-dose protocols demonstrates $40 \%, 21 \%$, and $55 \%$ reduction respectively in children $0-4,5-9$, and $10-16$ years of age (Fig 3 ).

\section{DISCUSSION}

In this study, we used the information obtained from the baseline cohort to implement age-specific pediatric neck CT protocols standardized across all scanners and facilities at our institution. Because most studies in the baseline sample were deemed of diagnostic quality by the reviewers, we decided to implement lower dose protocols using the first quartile $\mathrm{CTDI}_{\mathrm{vol}}$ for each age group 
๑Baseline ש Followup

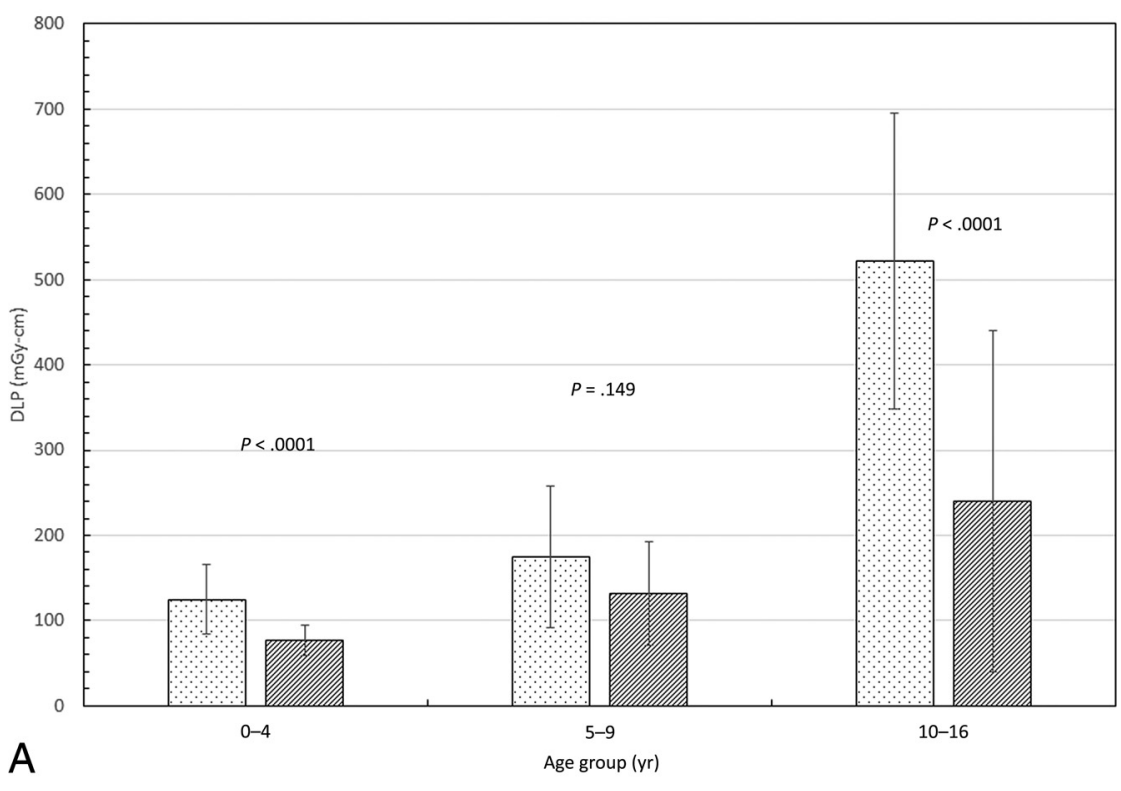

$\square$ Baseline $\square$ Followup

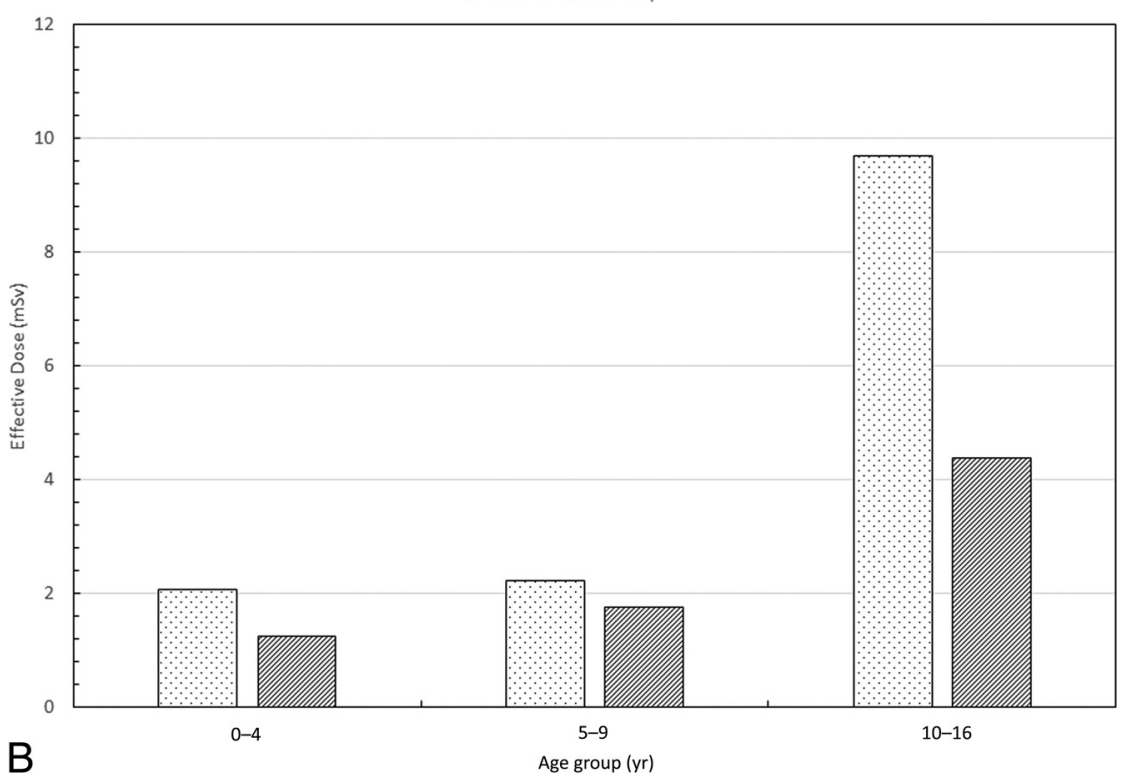

FIG 3. Comparison of the median values of the dose-length product $(A)$ and estimated effective dose $(B)$ between the 2 cohorts. Also shown are the $P$ values from an unpaired $t$ test.
Figure $2 A,-B$ shows a comparison of median values of the tube current and CT$\mathrm{DI}_{\mathrm{vol}}$ between the 2 cohorts. While the DLP dropped only moderately for the 2 youngest cohorts, there was a significant drop in the DLP for the oldest age group. The reduction in the median $\mathrm{CTDI}_{\mathrm{vol}}$ for the follow-up cohort was far more dramatic, $\sim 40 \%-65 \%$. Figure $2 C$ shows the median scan length for the 3 age groups. Note that there was no significant difference in the scan length between the baseline and follow-up cohorts for any of the age groups. This is important because these data reflect no attempt being made to "shorten" the length of the CT scan and risk missing clinically significant findings to simply reduce the dose.

Table 3 shows that the median scan length increased monotonically from $\sim 17$ to $27 \mathrm{~cm}$ across the 3 age groups for both cohorts. For the baseline cohort, the DLP changed from 125 to 175 mGy-cm (a factor of $\sim 1.4$ ) between the $0-4$ and the 5-9 age groups. However, there is a sharp rise in the DLP from 175 to $522 \mathrm{mGy}-\mathrm{cm}$ (a factor of $\sim 3$ ) between the 5-9 and the 10-16 age groups. One reason could be that the patients in the 0-4 and the 5-9 age groups were scanned at 80 or $100 \mathrm{kV}$, while the 10 - to 16 -year groups were scanned at 120 $\mathrm{kV} .^{21}$

For the follow-up cohort, the DLP changed from 77 to $132 \mathrm{mGy}-\mathrm{cm}$ (a factor of $\sim 1.7$ ) between the $0-4$ and 5-9 age groups. However, the DLP rose from 132 to $240 \mathrm{mGy}-\mathrm{cm}$ (a factor of $\sim 1.8$ ) between the 5-9 and the 10-16 age groups. This finding is in contrast to the increase in DLP by a factor of 3 in the baseline cohort. We believe this difference to be the result of better coaching as a reference. Our rationale for this choice was that the first quartile (25th percentile) of the CTDI ${ }_{\text {vol }}$ for each age group represents a reasonable compromise between the radiation dose and diagnostic quality for pediatric neck CT.

Data in Table 3 indicate that the median values of the tube current and CTDI ${ }_{\mathrm{vol}}$ increased from the youngest to the oldest age groups. This increase is to be expected and is a reflection of the use of automatic tube current modulation, which increases the radiation output to compensate for the increased patient size. ${ }^{13,20}$ For the baseline cohort, the median tube current varied by a factor of $\sim 1.8(150-266 \mathrm{mAs})$, while the CTDI ${ }_{\mathrm{vol}}$ increased by a factor of $\sim 3(7-20 \mathrm{mGy})$ from the youngest to the oldest age group. For the follow-up cohort, these parameters varied by a factor of $\sim 1.3(128-165 \mathrm{mAs})$ and $1.6(4.4-7$ $\mathrm{mGy}$ ), respectively. and re-education of the technologists, resulting in many of the scans $(\sim 35 \%)$ in this cohort being obtained at $100 \mathrm{kV}$.

Figure 4 shows that the median SNR and the image-quality scores were approximately the same (within $1 \mathrm{SD}$ ) across all age groups. The SNR not changing much is to be expected because larger patients were automatically scanned with higher kilovolt and/or tube currents to compensate for their size. Despite slight differences in the image-quality scores between the baseline and the follow-up cohorts for all 3 age groups, the median scores were well above 3 (making the scans diagnostically acceptable).

The estimated effective dose was reduced by $40 \%, 21 \%$, and $55 \%$ for the follow-up protocol compared with the baseline (Table 3 ). The estimated effective doses did not change much between the 2 youngest age groups (2.1-2.2 $\mathrm{mSv}$ for baseline and 1.2-1.7 $\mathrm{mSv}$ for the 
$\square$ Baseline $\square$ Followup

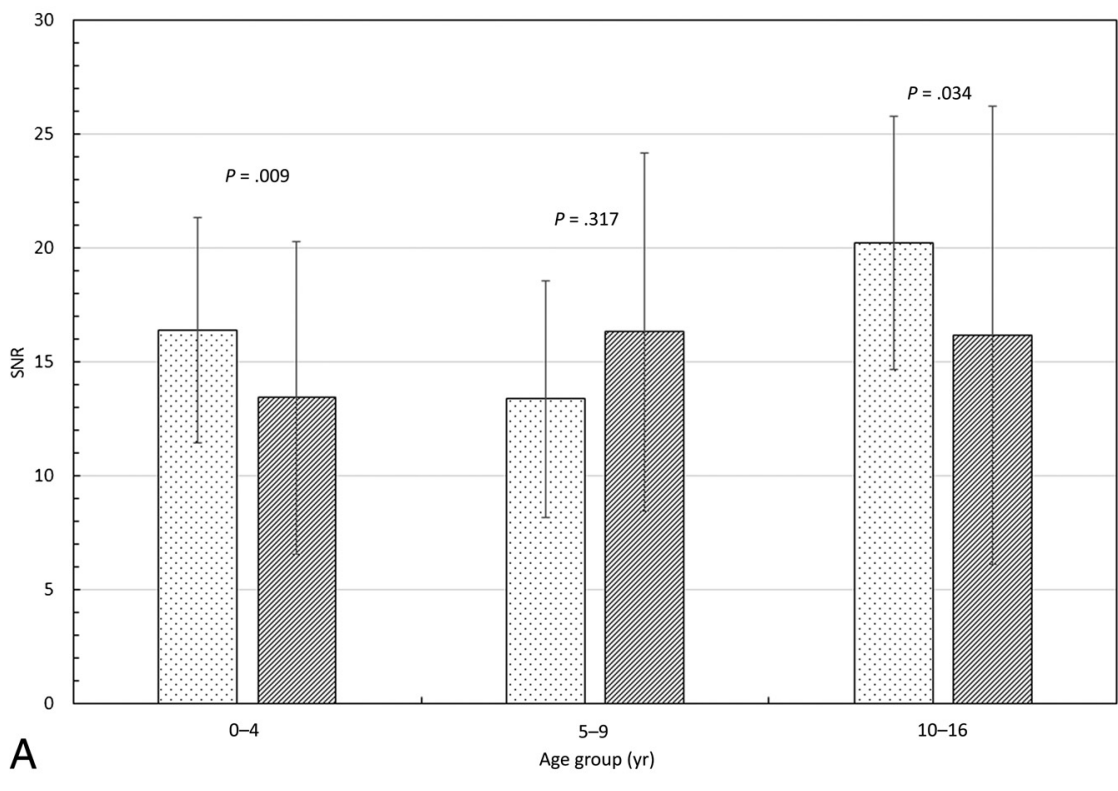

$\square$ Baseline Followup

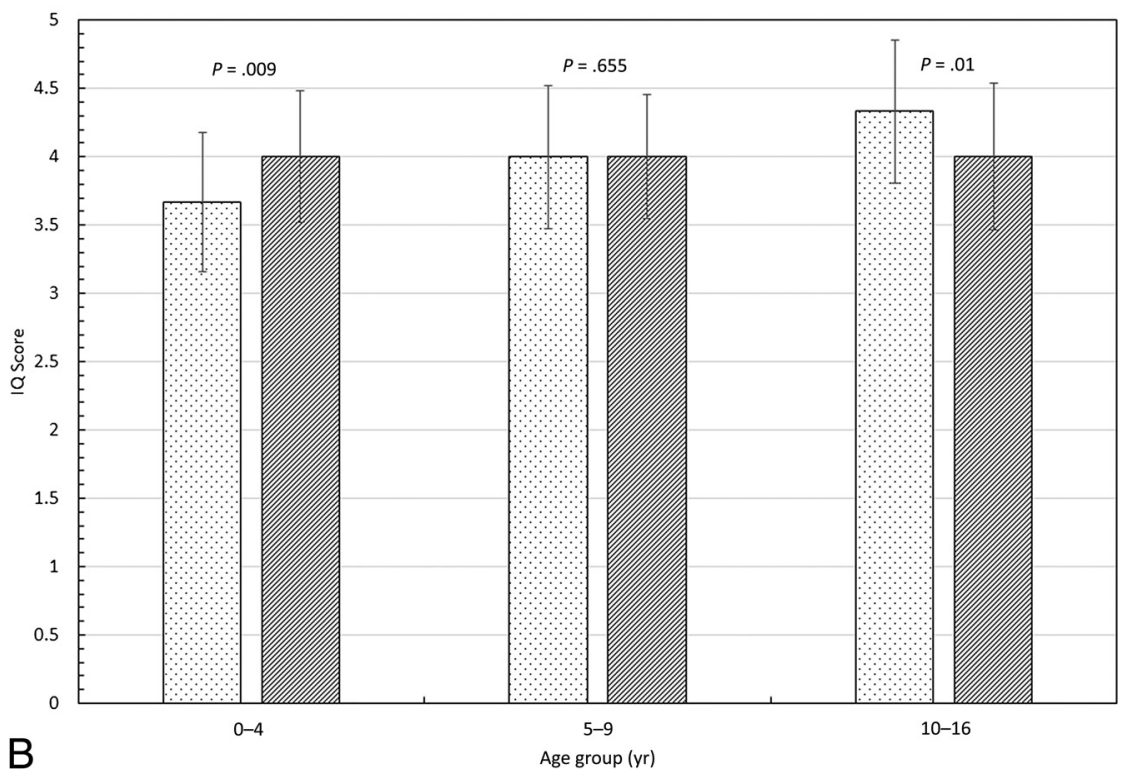

FIG 4. Comparison of the median values of the signal-to-noise ratio $(A)$ and image-quality score $(B)$ between the 2 cohorts. Also shown are the $P$ values from an unpaired $t$ test. reconstruction was performed using filtered back-projection algorithms. At the time of the study (2013-2016), we had not switched to iterative reconstruction, which is now routinely used on all our scanners. However, iterative reconstruction has its own challenges: Depending on what "level" of mixing one uses, the texture of the image can be vastly different between even identical scanners. With the use of newer, iterative reconstruction algorithms, it may be possible to reduce the radiation dose even further. Last, the effective doses listed in this study are fairly broad estimates, and individual patient doses could easily vary by up to a factor of $\geq 2$ depending on the body habitus. Thus, caution must be used in interpreting these results. However, we believe that this is a fair way of estimating effective doses from clinical scans for a "standard" patient, to design appropriate CT protocols.

\section{CONCLUSIONS}

The main inference to be drawn from this study is that even in a large clinical practice, with a well-reasoned choice of target $\mathrm{CTDI}_{\mathrm{vol}}$ and using techniques such as automatic tube current modulation, it is possible to optimize the amount of radiation used for performing CT examinations without compromising on the diagnostic image quality. While this is important for patients of all age groups, it is of particular importance for pediatric patients due to their increased radiation risk.

Disclosures: M. Vittoria Spampinato-UNRELATED: Grants/Grants: Bracco.* *Money paid to the institution. follow-up). However, the effective dose increased quite substantially when one looks at the oldest age groups. For the baseline cohort, the effective dose increased by a factor of $\sim 5(2.1-9.7 \mathrm{mSv})$ from the youngest to the oldest group. For the follow-up cohort, there was a similar increase by a factor of $\sim 4(1.2-4.4 \mathrm{mSv})$. We did not use the $\mathrm{k}$ conversion factors to estimate the effective dose for individual patients. ${ }^{22}$ Rather, as explained before, we used only the median value of the DLP and age for each subgroup for the estimation of the effective dose. While this makes the estimate somewhat broad, we believe it is still a good way of comparing the radiation risks between the 2 cohorts.

This study has certain limitations. First, all examinations were conducted on 64- and 128-slice Siemens scanners. Thus, the results may not necessarily be readily transferable to examinations conducted on scanners by other manufacturers. Second, all image

\section{REFERENCES}

1. Broder J, Fordham LA, Warshauer DM. Increasing utilization of computed tomography in the pediatric emergency department, 2000-2006. Emerg Radiol 2007;14:227-32 CrossRef Medline

2. Markel TA, Kumar R, Koontz NA, et al. The utility of computed tomography as a screening tool for the evaluation of pediatric blunt chest trauma. J Trauma 2009;67:23-28 CrossRef Medline

3. Blackwell CD, Gorelick M, Holmes JF, et al. Pediatric head trauma: changes in use of computed tomography in emergency departments in the United States over time. Ann Emerg Med 2007;49: 320-24 CrossRef Medline

4. Brenner DJ, Hall EJ. Computed tomography-an increasing source of radiation exposure. $N$ Engl J Med 2007;357:2277-84 CrossRef Medline

5. Shahi V, Brinjikji W, Cloft HJ, et al. Trends in CT utilization for pediatric fall patients in US emergency departments. Acad Radiol 2015;22:898-903 CrossRef Medline 
6. Brenner D, Elliston C, Hall E, et al. Estimated risks of radiationinduced fatal cancer from pediatric CT. AJR Am J Roentgenol 2001; 176:289-96 CrossRef Medline

7. UNSCEAR 2013 Report. Sources, Effects and Risks of Ionizing Radiation. 2013. http://www.unscear.org/docs/reports/2013/13-85418_ Report_2013_Annex_A.pdf. Accessed January 15, 2017

8. Brenner DJ. Estimating cancer risks from pediatric CT: going from the qualitative to the quantitative. Pediatr Radiol 2002;32:228-31; discussion 242-44 CrossRef Medline

9. Mettler FA Jr, Wiest PW, Locken JA, et al. CT scanning: patterns of use and dose. J Radiol Prot 2000;20:353-59 CrossRef Medline

10. Zacharias C, Alessio AM, Otto RK, et al. Pediatric CT: strategies to lower radiation dose. AJR Am J Roentgenol 2013;200:950-56 CrossRef Medline

11. Berrington de Gonzalez A, Mahesh M, Kim KP, et al. Projected cancer risks from computed tomographic scans performed in the United States in 2007. Arch Intern Med 2009;169:2071-77 CrossRef Medline

12. Miglioretti DL, Johnson E, Williams A, et al. The use of computed tomography in pediatrics and the associated radiation exposure and estimated cancer risk. JAMA Pediatr 2013;167:700-07 CrossRef Medline

13. Spampinato MV, Tipnis S, Tavernier J, et al. Thyroid doses and risk to paediatric patients undergoing neck CT examinations. Eur Radiol 2015;25:1883-90 CrossRef Medline

14. Yamauchi-Kawaura C, Fujii K, Aoyama T, et al. Radiation dose evaluation in head and neck MDCT examinations with a 6-year-old child anthropomorphic phantom. Pediatr Radiol 2010;40:1206-14 CrossRef Medline
15. Huda W, Spampinato MV, Tipnis SV, et al. Computation of thyroid doses and carcinogenic radiation risks to patients undergoing neck CT examinations. Radiat Prot Dosimetry 2013;156:436-44 CrossRef Medline

16. Paolicchi F, Faggioni L, Bastiani L, et al. Optimizing the balance between radiation dose and image quality in pediatric head CT: findings before and after intensive radiologic staff training. $A J R$ Am J Roentgenol 2014;202:1309-15 CrossRef Medline

17. Scholtz JE, Kaup M, Kraft J, et al. Objective and subjective image quality of primary and recurrent squamous cell carcinoma on head and neck low-tube-voltage $80-\mathrm{kVp}$ computed tomography. Neuroradiology 2015;57:645-51 CrossRef Medline

18. Gaddikeri S, Andre JB, Benjert J, et al. Impact of model-based iterative reconstruction on image quality of contrast-enhanced neck CT. AJNR Am J Neuroradiol 2015;36:391-96 CrossRef Medline

19. Deak PD, Smal Y, Kalender WA. Multisection CT protocols: sex- and age-specific conversion factors used to determine effective dose from dose-length product. Radiology 2010;257:158-66 CrossRef Medline

20. Kalra MK, Maher MM, Toth TL, et al. Techniques and applications of automatic tube current modulation for CT. Radiology 2004;233: 649-57 CrossRef Medline

21. Yu L, Bruesewitz MR, Thomas KB, et al. Optimal tube potential for radiation dose reduction in pediatric $C T$ : principles, clinical implementations, and pitfalls. Radiographics 2011;31:835-48 CrossRef Medline

22. The 2007 Recommendations of the International Commission on Radiological Protection: ICRP Publication 103. Ann ICRP 2007;37: 1-332 CrossRef Medline 